\title{
High-speed Si resonant cavity enhanced photodetectors and arrays
}

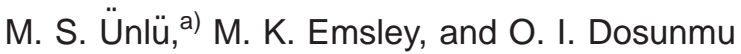 \\ Electrical and Computer Engineering, Boston University, 8 Saint Mary's Street, Boston, Massachusetts 02215 \\ P. Muller and Y. Leblebici \\ School of Engineering, Swiss Federal Institute of Technology, 1015 Lausanne, Switzerland
}

(Received 12 September 2003; accepted 15 December 2003; published 14 May 2004)

\begin{abstract}
Over the past decade a new family of optoelectronic devices has emerged whose performance is enhanced by placing the active device structure inside a Fabry-Perot resonant microcavity [P. E. Green, IEEE Spectrum 13 (2002)]. The increased optical field allows photodetectors to be made thinner and therefore faster, while simultaneously increasing the quantum efficiency at the resonant wavelengths. We have demonstrated a variety of resonant cavity enhanced (RCE) photodetectors in compound semiconductors [B. Yang, J. D. Schaub, S. M. Csutak, D. J. Rogers, and J. C. Campbell, IEEE Photonics Technol. Lett. 15, 745 (2003)] and Si [M. K. Emsley, O. I. Dosunmu, and M. S. Unlü, IEEE J. Selected Topics Quantum Electron. 8, 948 (2002)], operating at optical communication wavelengths ranging from $850 \mathrm{~nm}$ to $1550 \mathrm{~nm}$. The focus of this article is on $\mathrm{Si}$ photodetectors and arrays. High bandwidth short distance communications standards are being developed based on parallel optical interconnect fiber arrays to meet the needs of increasing data rates of interchip communication in modern computer architecture. To ensure that this standard becomes an attractive option for computer systems, low cost components must be implemented on both the transmitting and receiving end of the fibers. To meet this low cost requirement silicon based receiver circuits are the most viable option, however, high speed, high efficiency silicon photodetectors present a technical challenge. Commercially reproducible silicon wafers with a high reflectance buried distributed Bragg reflector (DBR) have been designed and fabricated [M. K. Emsley, O. I. Dosunmu, and M. S. Unlü, IEEE J. Selected Topics Quantum Electron. 8, 948 (2002)]. The substrates consist of a two-period, 90\% reflecting, DBR fabricated using a double silicon-on-insulator (SOI) process. Resonant-cavity-enhanced (RCE) Si photodetectors have been fabricated with $40 \%$ quantum efficiency at $850 \mathrm{~nm}$ and a FWHM of 29 ps suitable for $10 \mathrm{Gbps}$ data communications. Recently, $1 \times 12$ photodetector arrays have been fabricated, packaged, and tested with silicon based amplifiers to demonstrate the feasibility of a low cost solution for optical interconnects. (C) 2004 American Vacuum Society. [DOI: 10.1116/1.1647591]
\end{abstract}

\section{INTRODUCTION}

Optical interconnects will impact the future of not only the telecommunications industry, but also the entire computing industry. It may sound like a bold statement; the computing industry has been getting along quite well for decades, but a simple look at the numbers will tell the story. Figure 1 shows the growth in bandwidth for CPU's in comparison to the speed of the peripheral bus. It is clear that for the past two decades a chasm has been opening between the two. One can consider the processing power of a computer to be inherently serial in nature, combining input/output (I/O) and CPU speeds. When I/O lags CPU speed by an order of magnitude, the computational throughput of the computer is no longer determined by the CPU. In this situation, the CPU becomes a commodity within computer architecture, which is clearly something that major microprocessor manufacturers have strived to avoid for many years.

The telecommunications industry has made huge investments in the long-distance high bandwidth links that can carry terabits of information, but fiber to the home remains elusive and many people still use dial-up modem access. An

${ }^{a}$ Electronic mail: selim@bu.edu interesting point is that data can be sent 3000 miles across the Atlantic Ocean in less time then it takes to go the $3 \mathrm{in}$. from the memory to the processor. Paul Green wrote in the December issue of IEEE Spectrum, "Both computers and the common carriers' systems run at multiple tens of gigabits per second. Dial-up modems carry, at best, $50 \mathrm{~kb} / \mathrm{s}$ - nowhere near enough to support the innovative new services on which the future prosperity of both the telecom and computer industries depends." 1

What is needed is a high speed data link for short haul (very short reach) communications. There are several standards being developed to satisfy the short haul communication traffic jam. These optical interconnects are based on arrays of laser transmitters coupled with similar arrays of photodetector receivers coupled through low cost plastic optical fiber arrays. One such standard being developed is Infiniband based on a $12 \times 1$ array of parallel optical interconnects operating at $2.5 \mathrm{Gbps}$ per channel.

Since there are many more downstream computers than upstream nodes there is an intense effort to develop low cost components. Owing to the low cost drive, $850 \mathrm{~nm}$ light sources are utilized due to the low cost of plastic optical fiber as well as the lower cost of $850 \mathrm{~nm}$ vertical cavity surface 


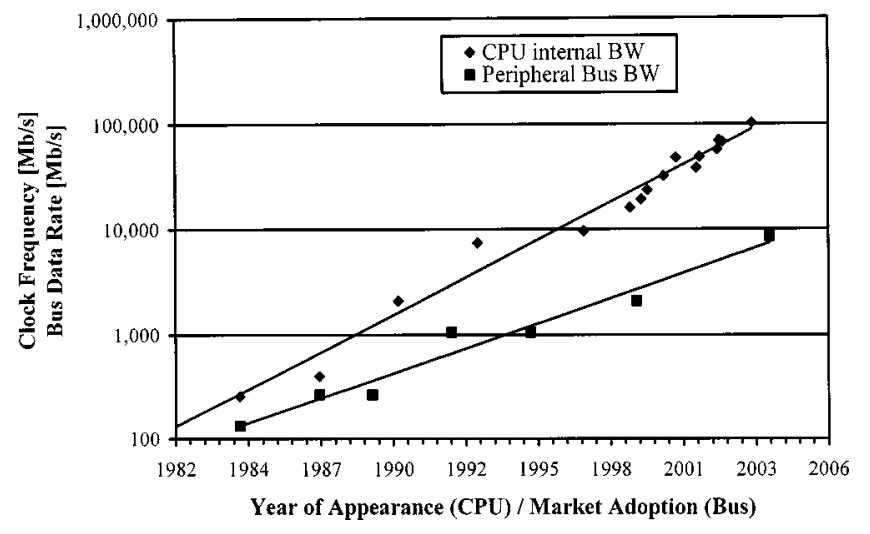

FIG. 1. CPU and peripheral bandwidth increase over time in the last 20 years.

emitting lasers (VCSEL). On the receiving end, III-V semiconductors are the current standard due to the optimum efficiency, but a serious effort to develop low cost alternatives has not been pursued until recently. One reason for this is that the relative cost of the photodetector compared to the laser has focused the effort towards reducing cost of the more expensive lasers. Market conditions have recently arisen, however, that make finding lower cost alternatives attractive in both the transmitters and the receivers.

There have been significant efforts to develop Si based photodetectors. ${ }^{2}$ It has been the standard in semiconductors that silicon based devices have always been lower in cost. The reason for this is quite simple; InP, currently the standard for high speed photodiodes, is available in wafer sizes typically 3 in. in diameter, while Si wafers are available in 12 in. diameters. This corresponds to a square millimeter cost of a few orders of magnitude less for Si than InP. There are also the fabrication costs which are inherently less for Si not only due to the overwhelming size of the Si processing industry, but also due to the simple fact that fewer wafers have to be fabricated because of the difference in area. Unfortunately for the industry, silicon exhibits poor optical absorption efficiency due to its indirect band gap, thus requiring long absorption path lengths. The long absorption length adversely affects the device bandwidth as in a typical device the contacts sandwich the absorption region, and carriers generated in this region must traverse to the contacts. The longer the distance between the contacts the longer it takes for all the carriers to be collected. Table I shows a comparison of com- mercially available InGaAs/InP based photodetectors and a $\mathrm{Si}$ photodetector. Also listed in Table I is the Si-resonant cavity enhanced (RCE) photodetector that has been fabricated in this work.

As is shown in Table I standard Si photodetectors have lower responsivity and much lower bandwidth and would be unable meet the specifications of the optical interconnect standards. It can also been seen however, that Si-RCE photodetectors come quite close in performance, and have an order of magnitude lower dark current, which is a typical advantage of Si over III-V devices.

It is prudent to stress the following points: (1) $\mathrm{Si}$ based photodetectors offer the ability to monolithically integrate detectors and receivers. (2) Standard Si photodetectors offer inadequate device performance. (3) Si-RCE photodetectors offer desirable performance. Below we describe the fundamentals of RCE photodetectors and discuss our experimental results on Si devices.

\section{RESONANT CAVITY ENHANCED PHOTODETECTORS}

Resonant cavity enhanced (RCE) photodetectors have been shown to provide the required bandwidth-efficiency product but have remained a challenge to reproduce through commercially available fabrication techniques. We have previously developed a method to produce silicon wafers with high reflectance buried distributed Bragg reflectors (DBR)., The substrates consist of a two-period, $90 \%$ reflecting, DBR fabricated using a double silicon-on-insulator (SOI) process. Discrete Si-RCE photodetectors have previously been fabricated with $40 \%$ quantum efficiency at $860 \mathrm{~nm}$, an impulse response FWHM of $25 \mathrm{ps}$, and a $3 \mathrm{~dB}$ bandwidth in excess of $10 \mathrm{GHz}^{5}$

An important figure of merit for high speed photodetectors is the bandwidth efficiency (BWE) product. That is, the transit time of the photogenerated carriers must be kept to a minimum while the absorption length must be sufficiently long so that a reasonable number of photons are absorbed and, in turn, carriers generated. One way to circumvent this problem involves fabricating the photodetector within a Fabry-Perot cavity. Fabry-Perot cavities are formed by sandwiching a region between two reflecting surfaces, where light incident perpendicular to the cavity results in spectrally dependent constructive and destructive interference of the electric field inside the cavity. The inherent benefit of the

TABLE I. Parameters for commercially available InGaAs and Si high speed photodetectors and results from our Si-RCE photodiodes.

\begin{tabular}{lcccc}
\hline \hline \multicolumn{1}{c}{ Parameter } & Unit & InGaAs $(70 \mu \mathrm{m})^{\mathrm{a}}$ & $\mathrm{Si}^{\mathrm{b}}$ & Si-RCE $(70 \mu \mathrm{m})$ \\
\hline Responsivity, $R$ & $\mathrm{~A} / \mathrm{W}$ & $0.45(\lambda=830 \mathrm{~nm})$ & $0.35(\lambda=780 \mathrm{~nm})$ & $0.32(\lambda=780 \mathrm{~nm})$ \\
Dark current, $I_{D}$ & $\mathrm{nA}$ & 10 & 0.02 & $3 \mathrm{pA}$ \\
Capacitance, $C$ & $\mathrm{pF}$ & 0.5 & 2 & 0.2 \\
Bandwidth, $B$ & $\mathrm{GHz}$ & 5 & $<1$ & 3.5 \\
Bias voltage, $V^{+}$ & $\mathrm{V}$ & 2 & 2 & 2 \\
\hline \hline
\end{tabular}

${ }^{\mathrm{a}}$ Reference 1 .

${ }^{\mathrm{b}}$ Reference 2 . 


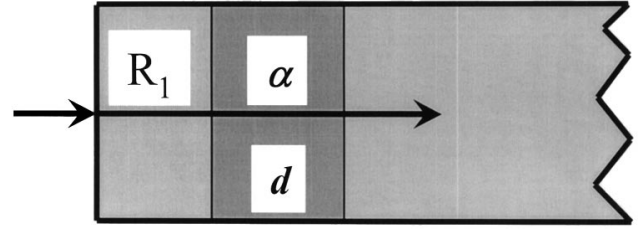

FIG. 2. Cross section of conventional single pass photodiode.

resonant cavity enhanced (RCE) structure is to increase the bandwidth-efficiency product over a conventional photodetector. The quantum efficiency $\eta$ for a conventional detector, as illustrated in Fig. 2, is governed by the optical absorption $\alpha$ of the semiconductor material, when the recombination current is negligible, given by ${ }^{6}$

$$
\eta=\left(1-R_{1}\right) \cdot\left(1-e^{-\alpha d}\right),
$$

where $R_{1}$ is the reflectivity of the top surface and $d$ is the thickness of the absorption region.

For semiconductors with low absorption coefficients, thick absorption regions are required to achieve high $\eta$ limiting the bandwidth of photodetectors. A cross-sectional illustration of a typical RCE photodetector can be seen in Fig. 3.

The constructive interference results in an increase of the electric field amplitude at specific points within the cavity over a limited spectral range. Since high-speed optical communications typically rely on narrow linewidth laser sources, fabricating photodetectors within a Fabry-Perot cavity is an ideal solution. For a given absorption length, the quantum efficiency of the detector over a specific wavelength range is increased, while the transit time and bandwidth remains constant as compared to a conventional photodiode. This results in an overall increase in the bandwidth efficiency product for the RCE photodiode. The peak quantum efficiency for a RCE photodetector is given by ${ }^{7}$

$$
\eta_{\max }=\left\{\frac{\left(1+R_{2} e^{-\alpha d}\right)}{\left(1-\sqrt{R_{1} R_{2}} e^{-\alpha d}\right)^{2}}\right\} \times\left(1-R_{1}\right) \cdot\left(1-e^{-\alpha d}\right),
$$

where $R_{2}$ is the reflectivity of the buried mirror.

Typical resonant cavity photodetectors are fabricated from compound semiconductor based materials by molecular beam epitaxy (MBE). For example AlAs/GaAs distributed Bragg reflectors (DBR) are lattice matched to GaAs and achieve high reflectivity using greater than 10 periods. RCE

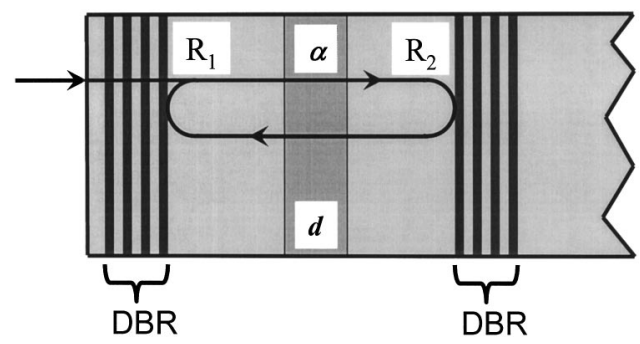

FIG. 3. Cross section of Fabry-Perot cavity with distributed Bragg reflectors.
Schottky GaAs-based photodetectors have been demonstrated with bandwidths in excess of $50 \mathrm{GHz}$ and peak quantum efficiency of $75 \% .^{8-10}$

Numerous attempts have been made to fabricate Si RCE photodetectors. ${ }^{11}$ Earlier devices utilized Si device structures deposited on top of dielectric mirrors. Various attempts included CVD as well as MBE, ${ }^{12}$ which resulted in a polycrystalline Si device layer. Photodiodes fabricated on this device layer typically suffered from high dark currents. Schaub et al. ${ }^{13}$ has reported Si RCE photodiodes with low dark currents that achieved a bandwidth in excess of $34 \mathrm{GHz}$ - the highest speed recorded for Si $p-i-n$ photodiodes. These RCE structures used a merged epitaxial layer overgrowth (MELO) process to form the absorption region on top of the buried DBR. Although this growth process is not a commercially viable technique, the results in themselves are significant.

Making use of silicon on insulator technology (SOI), silicon-based RCE photodiodes have also been developed. ${ }^{14}$ While there have been reports of multilayer silicon-oninsulator (SOI) wafers, ${ }^{15}$ there have been few efforts on purposely manufactured reflecting substrates. Ishikawa et al. ${ }^{16,17}$ used a combination of separation by implantation of oxygen (SIMOX) and epitaxy to develop a DBR with a peak reflectance of $90 \%$. The DBR used 5 periods of $\mathrm{Si} / \mathrm{SiO}_{2}$ which were created using SIMOX to produce the buried oxide layer and molecular beam epitaxy (MBE) to grow the Si layers. In contrast to SIMOX, using the-ion-cut process it is viable to develop commercially reproducible $\mathrm{Si}$ wafer with a high reflectance buried DBR (Ref. 4) for $\mathrm{Si}$ RCE optoelectronics. ${ }^{18,19}$ These wafers, fabricated by a repeated SOI process, have buried DBR structures with reflectivity in excess of $90 \%$ using only a two-period $\mathrm{Si} / \mathrm{SiO}_{2}$ structure and have an epitaxy ready single crystalline surface. With these double-SOI reflecting substrates, not only can Si RCE photodetectors be a commercial reality but also, due to the ubiquitous nature of the silicon industry, a wide array of other applications such as wavelength sensors, RCE photodetector arrays, as well as on chip signal conditioning become possible.

\section{PHOTODETECTOR FABRICATION}

Si-RCE $p-i-n$ photodetectors were fabricated in the epitaxial device layer, which was approximately $2.1 \mu \mathrm{m}$ in total thickness, using standard Si device fabrication techniques. The device structure is shown schematically in Fig. 4. The structure has a buried $n^{+}$implant of $1 \times 10^{19} \mathrm{~cm}^{-3}$ As concentration and a $p^{+}$implant of $1 \times 10^{19} \mathrm{~cm}^{-3} \mathrm{~B}$ concentration on the surface, while the epitaxial Si layer is left undoped yielding the vertical $p-i-n$ diode. Below the device layer is the two period DBR creating a Fabry-Perot cavity, with the air/Si interface acting as the top reflector.

In this paper we present $\mathrm{Si}-\mathrm{RCE} 12 \times 1$ photodetector arrays that have been fabricated and packaged with silicon based amplifiers to demonstrate the feasibility of a low cost monolithic silicon photoreceiver array. The $12 \times 1$ photodetector arrays were designed for the PAROLI product line specification by Infineon. ${ }^{20}$ The specification calls for 12 


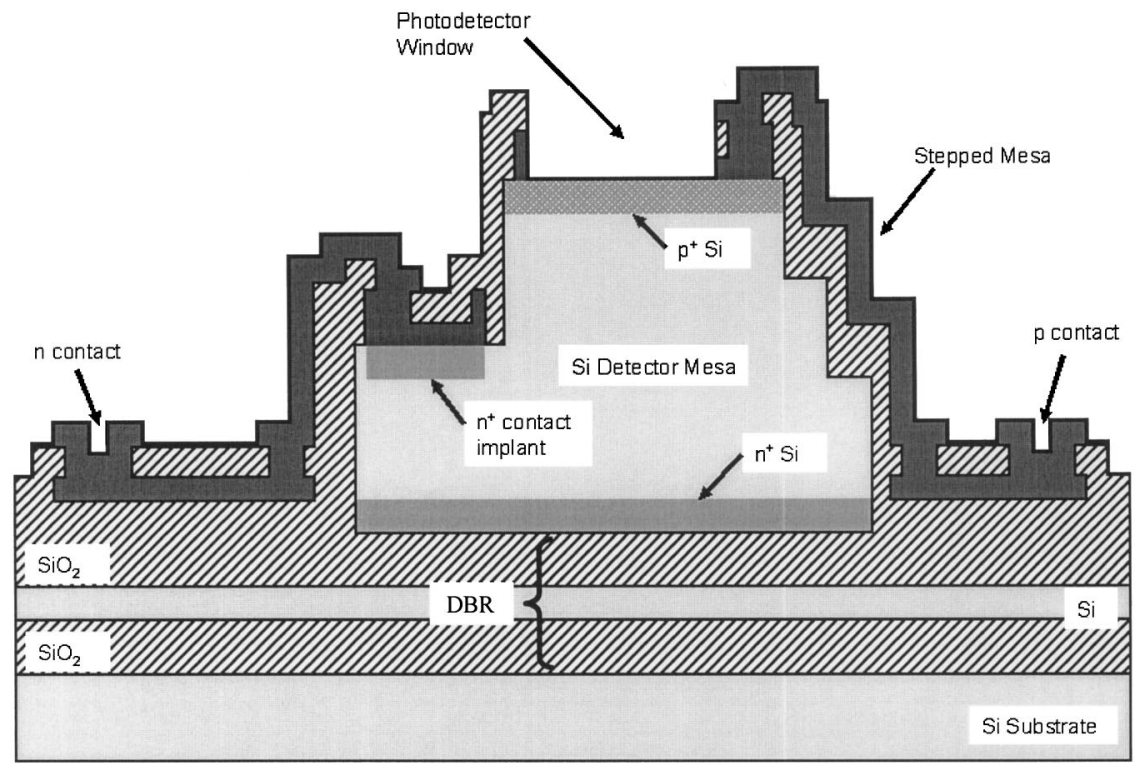

FIG. 4. Cross section of Si-RCE $p-i-n$ photodetector.

photodetectors with between $30 \mu \mathrm{m}$ and $80 \mu \mathrm{m}$ diam photodetector active area in a linear array on a $250 \mu \mathrm{m}$ pitch. The fabricated $12 \times 1$ photodetector array can be seen in Fig. 5 .

\section{RESULTS AND DISCUSSION}

\section{A. I- $V$ characteristics}

After fabrication, the photodetectors' $I-V$ characteristics were measured using an HP4156A Parameter Analyzer. $I-V$ measurements were done on two rows of photodetectors randomly selected from one wafer. These discrete photodetectors range in diameter from $22 \mu \mathrm{m}$ to $200 \mu \mathrm{m}$. The $I-V$ characteristic of the $200 \mu \mathrm{m}$ diam photodetector can be seen in Fig. 6.

Excellent reverse voltage characteristics were observed with all devices having less than $10 \mathrm{nA}$ of current with $35 \mathrm{~V}$ of applied bias. The reverse voltage characteristic for the 200 $\mu \mathrm{m}$ diam photodetector can be seen in Fig. 7.

The dark current on $200 \mu \mathrm{m}$ diam photodetectors was measured as $9 \mathrm{pA}$ or $29 \mathrm{nA} / \mathrm{cm}^{2}$ at reverse bias of $1.8 \mathrm{~V}$, which is the bias voltage used in receiver testing discussed later. On $22 \mu \mathrm{m}$ diam devices, the dark current density measured as $105 \mathrm{nA} / \mathrm{cm}^{2}$ at the same bias level. The increase in dark current density with decreasing device size (increasing periphery to area ratio) can be explained by increased contribution of surface recombination current.

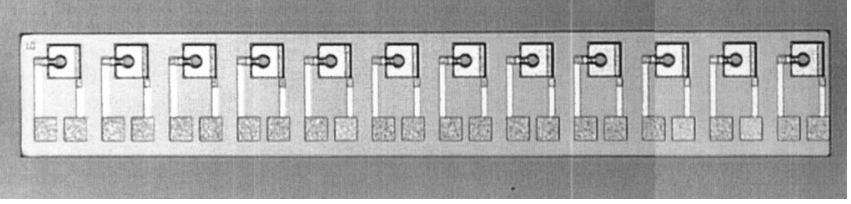

FIG. 5. Parallel $12 \times 1$ photodetector array.

\section{B. Quantum efficiency}

Spectral quantum efficiency measurements were performed using a tunable Ti:sapphire laser source and a calibrated, NIST traceable, Si photodetector with known spectral responsivity for photocurrent normalization. A reference scan was performed with the calibrated Si photodetector to determine the optical power at the test point as a function of wavelength. Measurement techniques are discussed in detail in earlier publications. ${ }^{21}$ It can be seen from Fig. 8 that the spectral responsivity agrees well with the simulation and that the responsivity near $822 \mathrm{~nm}$ is approximately $0.30 \mathrm{~A} / \mathrm{W}$, which is roughly $40 \%$ quantum efficiency. It is also evident in Fig. 8 that the peak wavelength does not occur at $850 \mathrm{~nm}$, which is due to a slightly thick top Si layer. Spectral tuning can be achieved by recessing the top Si thickness, ${ }^{7}$ although

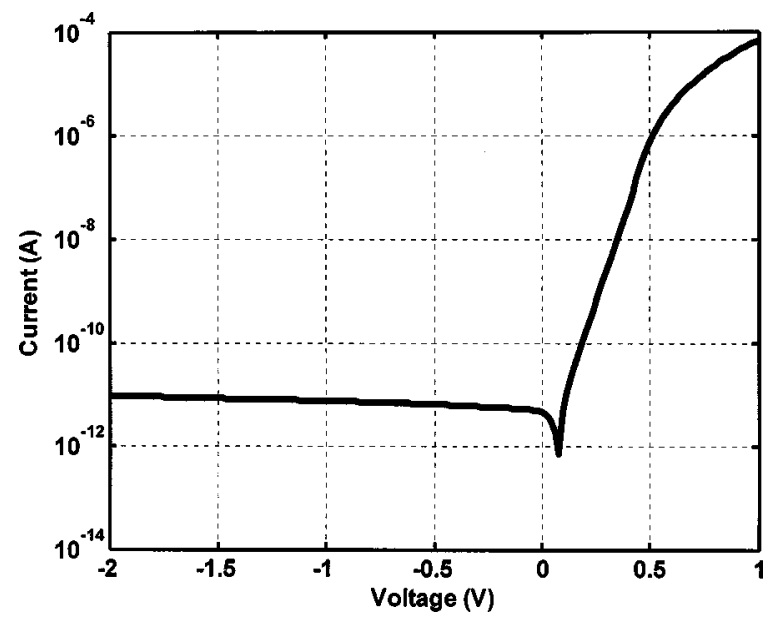

FIG. 6. $I-V$ characteristic for $200 \mu \mathrm{m}$ diam device. 


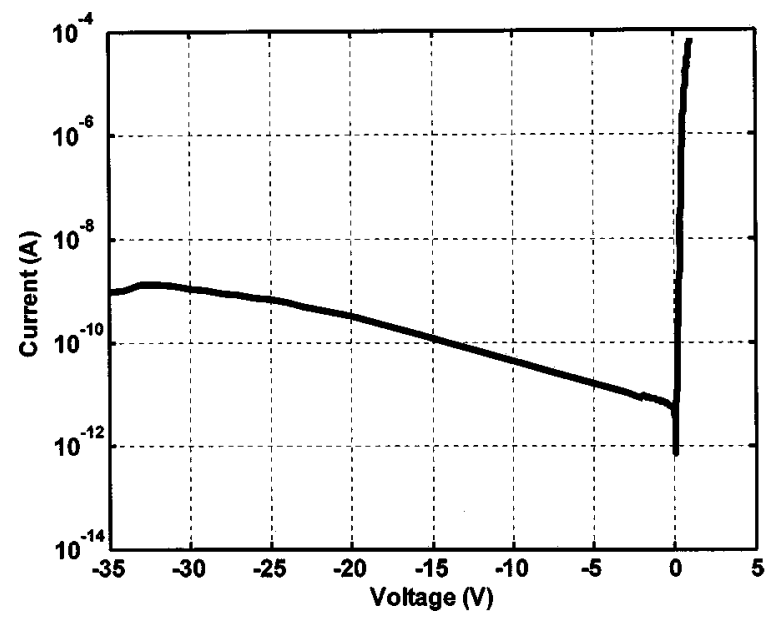

FIG. 7. $I-V$ curve for $200 \mu \mathrm{m}$ diam device showing current increase as a function of bias.

this was not performed during this study. The discrepancy between the measured and simulated responsivity peaks is presumed to be measurement error.

\section{Temporal response}

High-speed characterization was performed on a microwave probe station using a $50 \mathrm{GHz}$ digitizing oscilloscope. A Ti:sapphire picosecond laser was used to perform impulse response measurements on the photodetectors. Figure 9 shows the temporal response obtained at $3 \mathrm{~V}$ reverse bias from a $22 \mu \mathrm{m}$ diam photodetector with measured FWHM value of 24.8 ps. The FWHM of 24.8 ps suggests a bandwidth above $10 \mathrm{GHz}$, which is well beyond the requirements for $10 \mathrm{Gbps}$ data communications.

\section{Frequency response}

To study the frequency response, measured temporal response data for various size devices at different bias values. Time domain measurements were converted to frequency domain using fast Fourier transform (FFT) as shown in Fig. 10.

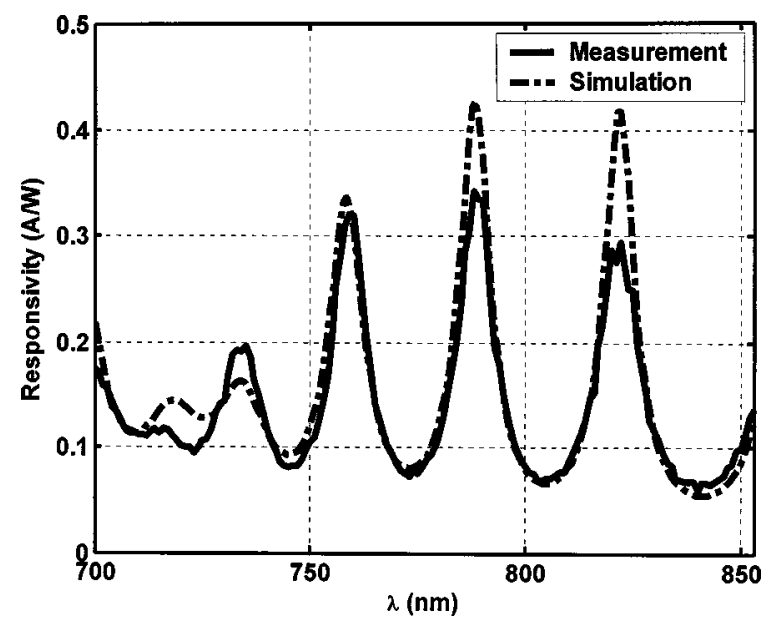

FIG. 8. Spectral quantum efficiency of RCE $p-i-n$ photodetector.

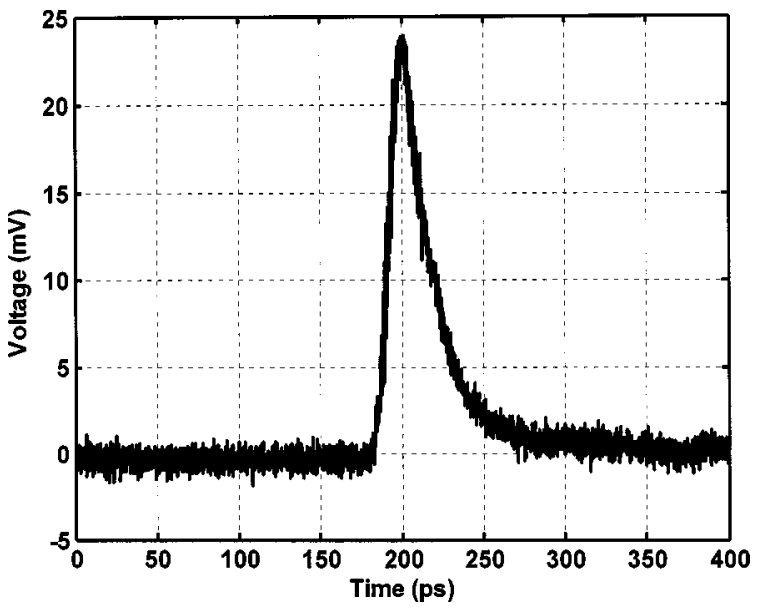

Fig. 9. Temporal response obtained at $3 \mathrm{~V}$ reverse bias from a $22 \mu \mathrm{m}$ diam photodetector with measured FWHM value of $24.8 \mathrm{ps}$. The device had a rise time of $12 \mathrm{ps}$ and a fall time of $44 \mathrm{ps}$.

A 4096 point FFT was performed on the sampled data. Figure 10 shows the $3 \mathrm{~dB}$ bandwidth obtained from a $22 \mu \mathrm{m}$ diam photodetector at $4 \mathrm{~V}$ reverse bias. Temporal responses were measured at different reverse bias voltage to determine the $3 \mathrm{~dB}$ frequency dependence on bias voltage. Devices were tested with bias voltage ranging from $0 \mathrm{~V}$ to $10 \mathrm{~V}$ in 1 $\mathrm{V}$ increments. Figure 11 shows the $3 \mathrm{~dB}$ frequency as a function of voltage for two randomly selected $22 \mu \mathrm{m}$ diam photodetectors.

\section{E. Bit error rate measurements}

Several samples were prepared for packaging with Helix HXR2312 3.6 GHz 12 channel receiver arrays. The samples were sent to Helix in Zurich, Switzerland and were bonded to the Helix receiver array using $25 \mu \mathrm{m}$ gold wire wedge bonding. There were limitations in using these amplifiers. The first and perhaps most important limitation is the mismatch input impedance of the Helix amplifier. The Helix amplifier is designed to have a matched capacitive load of

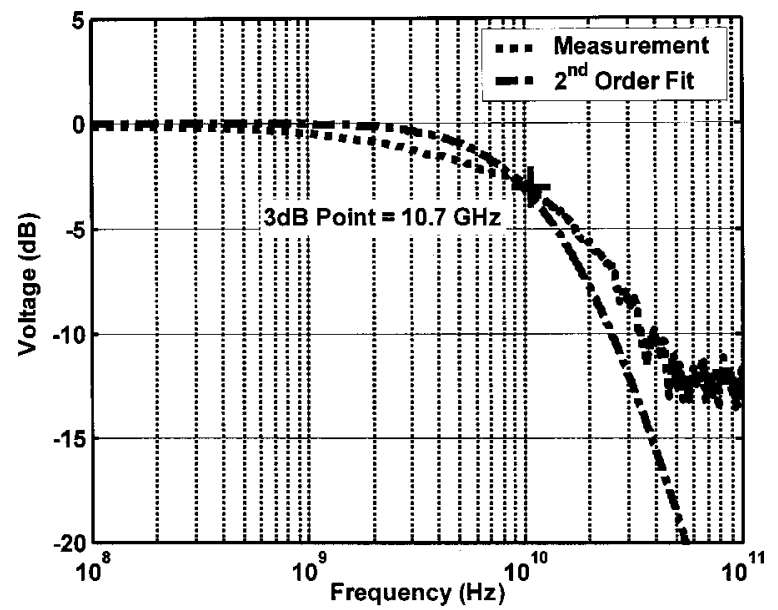

FIG. 10. FFT of temporal response for the $22 \mu \mathrm{m}$ diam photodiode at $4 \mathrm{~V}$ reverse bias. 


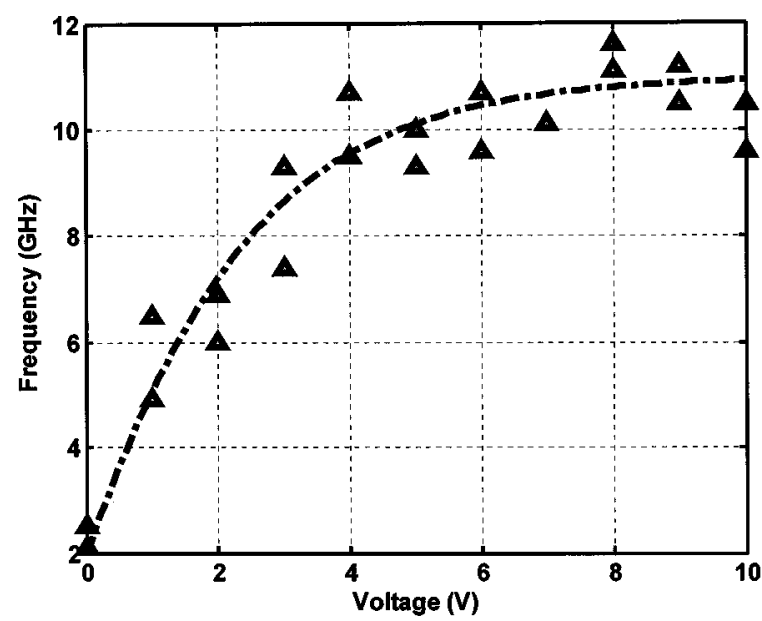

FIG. 11. $3 \mathrm{~dB}$ bandwidth as a function of bias voltage for two $22 \mu \mathrm{m}$ diam photodiodes (with trend line).

$450 \mathrm{fF}$, while our photodetectors have a $70 \mathrm{fF}$ capacitance. One other draw back is that even though the specification mentioned earlier called for $30-80 \mu \mathrm{m}$ diam photodetectors the testing was done with $50 \mu \mathrm{m}$ core multimode fiber (MMF). This means that the $42 \mu \mathrm{m}$ photodetector was only capturing $70 \%$ of the incident light at best.

The photodetectors were tested with the Helix receiver to measure the bit error rate BER and the eye-diagram. The photoreceiver was tested using an HP8664A synthesized signal generator connected to a HP70841B pattern generator which was connected to a laser driver and an $850 \mathrm{~nm} 10 \mathrm{GHz}$ laser diode, which was coupled to MMF and sent through an EXFO FVA-3100C variable attenuator. The receiver is then connected to an HP70842B error detector to measure BER and an Agilent infinium DCA wide bandwidth oscilloscope with HP54743A TDR module to measure the eye-diagram. During the test the photodetector is biased at $1.8 \mathrm{~V}$ reverse bias.

The pattern generator was setup to send a PRBS $2^{23}-1$ sequence to laser diode with a clock frequency of $1 \mathrm{GHz}, 1.5$ $\mathrm{GHz}, 2 \mathrm{GHz}$, and $3 \mathrm{GHz}$ on four separate runs. BER was measured using the HP70842B error detector which had the same pseudo random bit sequence (PRBS) $2^{23}-1$ programmed for comparison. Figure 12 shows the measured BER from a randomly selected single detector in a $12 \times 1$ array. Ideal "error free" transmission is considered to be 1 part in 1 trillion or a BER of $10^{-12}$. At $10 \mathrm{GHz}$ aggregate speed the Si-RCE array was able to achieve a BER of $10^{-12}$ at $-12 \mathrm{dBm}$.

\section{F. Eye diagram measurements}

Eye diagrams are used to measure the jitter in a received signal which can be described as the high frequency fluctuation of the signal from its ideal position. For communication standards masks are defined for the eye diagrams that declare regions off limits, and if the signal should fall within the region then the device would fail the standards certification. Typically eye diagrams are measured on a photodetector con-

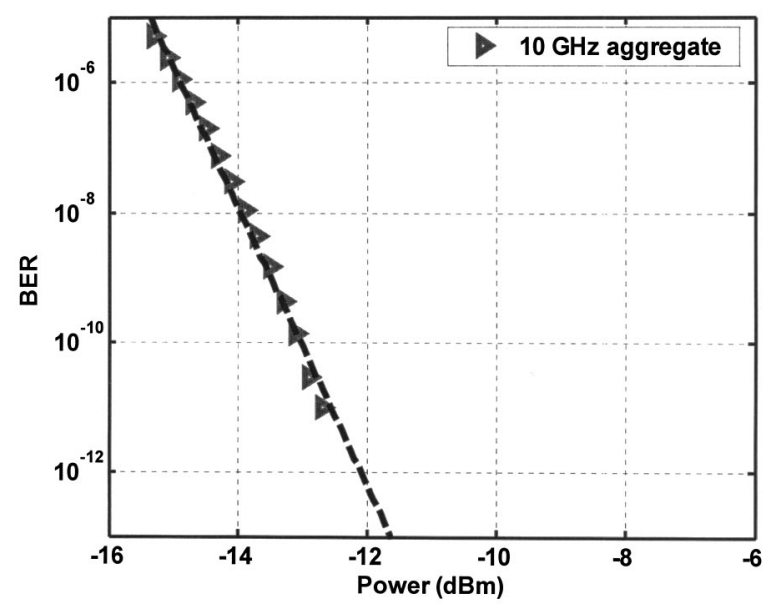

FIG. 12. Bit error rate measurement on a single detector in a $12 \times 1$ photodetector array.

nected to a transimpedance amplifier with a fixed gain. Therefore at higher frequencies a lowering of the output magnitude will occur due to $3 \mathrm{~dB}$ roll off. The Helix HXR2312 has a rail-to-rail limiting amplifier built in which means the magnitude of the output is constant for changing input magnitudes. Therefore the usefulness of the eye diagrams is questionable in this study as it can only reasonably measure the lateral eye opening due to diffusing carriers. Figure 13 shows the eye-diagram for the same randomly selected detector in a $12 \times 1$ array used in the BER results. The eye shows good opening at an aggregate speed greater than $10 \mathrm{GHz}$ and $-12.7 \mathrm{dBm}$ optical power. The plot does not show any characteristic signs of long diffusion tails.

\section{CONCLUSIONS AND FUTURE WORK}

This work has presented commercially reproducible $\mathrm{Si}$ substrates with buried distributed Bragg reflectors, having reflectivity over $90 \%$, used to fabricate Si-RCE $p-i-n$ photodetectors capable of responsivity above $0.3 \mathrm{~A} / \mathrm{W}$ and band-

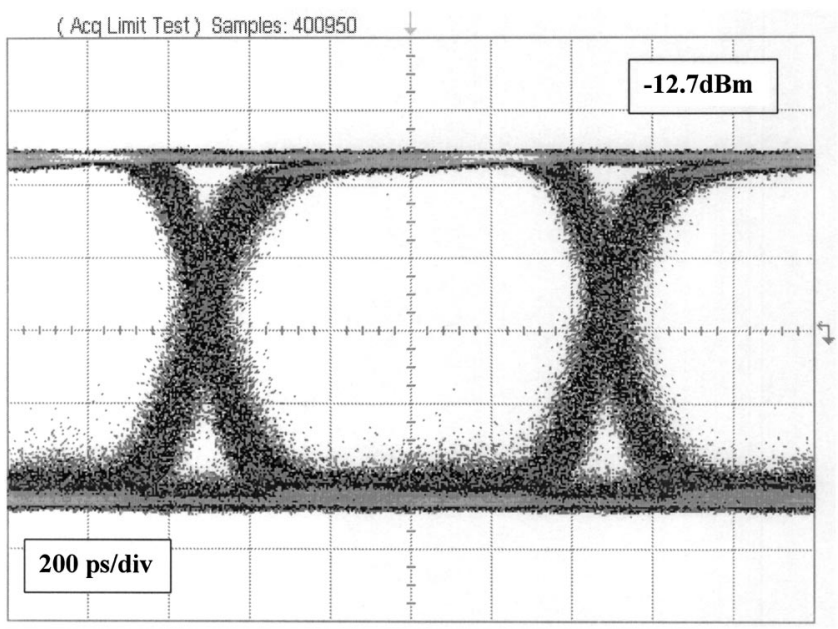

FIG. 13. Eye diagram for a single photodetector in a $12 \times 1$ array at an aggregate speed greater than $10 \mathrm{GHz}$. 


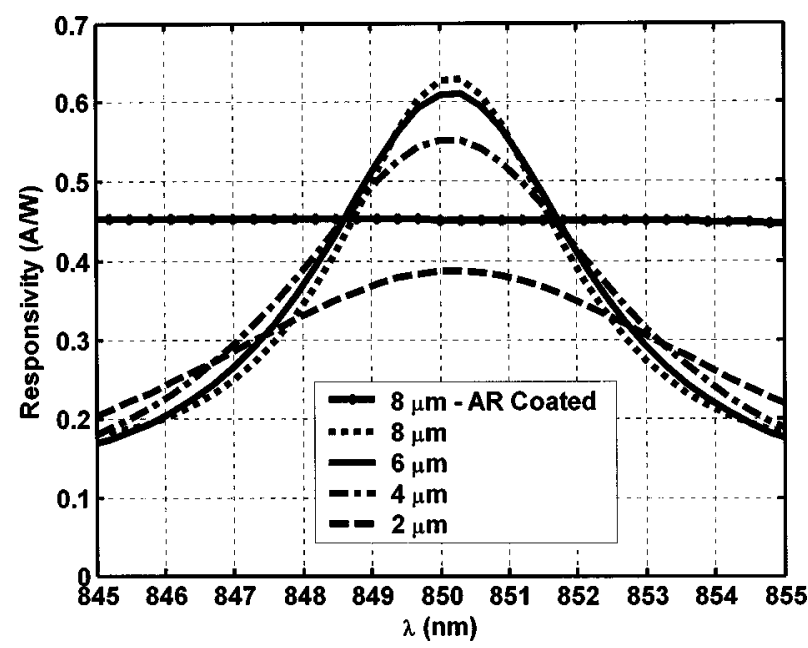

FIG. 14. Simulated responsivity for RCE photodetectors of different absorption lengths as well as $8 \mu \mathrm{m}$ "two-pass" photodetector.

width in excess of $10 \mathrm{GHz}$. Photodetector arrays were fabricated and wire bonded with existing $\mathrm{Si}$ based receiver circuits to make an all-Si photoreceiver operating at $3 \mathrm{GHz}$, showing performance that could soon compete with existing compound semiconductor photodetectors for a fraction of the cost. The wafers could also be used to fabricate a host of $\mathrm{Si}$ based integrated optoelectronics owing to the availability of Si processing and the availability of large wafer sizes. These wafers are well suited for large scale integration and are compatible with standard CMOS processing making them ideal for fabricating photodetectors monolithically with receiver circuits.

The benefit of a RCE structure is to increase the efficiency for a given absorption thickness detector while maintaining the bandwidth. The drawback of the RCE structure, however, is the wavelength selectivity of the photodetector efficiency. To counteract this selectivity and yet still benefit from the RCE structure one can coat the top surface of the detector with an antireflection (AR) coating which will result in a "two-pass" detector where the light enters the photodetector and reflects off the buried mirror resulting in two passes of the absorption length. This will increase efficiency over a conventional detector by nearly twofold while keeping the wavelength insensitivity over a substantial range. Figure 14 shows the simulated responsivity-wavelength dependence of different absorption thicknesses as well as an $8 \mu \mathrm{m}$ thickness with an AR coating showing the wavelength insensitivity around $850 \mathrm{~nm}$. At this thickness, a two-pass Si photodiode is not wavelength selective and has comparable efficiency to compound semiconductor alternatives. The $3 \mathrm{~dB}$ bandwidth of an $80 \mu \mathrm{m}$ diam detector with $1.8 \mathrm{~V}$ reverse bias is estimated to be about $4 \mathrm{GHz}$ thus satisfying the efficiency and bandwidth requirements for $40 \mathrm{~Gb} / \mathrm{s}$ aggregate speed $1 \times 12$ interconnects.

\section{ACKNOWLEDGMENTS}

The authors wish to thank Mike Ameen and Mark Harris at Axcelis Technologies for providing ion-implantation ser- vices for this project. We would also like to thank Philippe Flückiger and the entire staff at the Center of MicroNanotechnology at EPFL for help in the fabrication of these detectors. Additionally we would like to thank Bruno Ghyselen of SOITEC SA for fabrication of the double SOI wafers. The authors also thank Martin Bossard and Jörg Wieland of Helix AG for packaging our detectors with their receiver circuits as well as for doing the BER and eyediagram testing. This research was sponsored by the Army Research Laboratory (ARL) and was accomplished under the ARL Cooperative Agreement No. DAAD17-99-2-0070. The views and conclusions contained in this document are those of the authors and should not be interpreted as representing the Laboratory or the U.S. Government.

${ }^{1}$ P. E. Green, IEEE Spectrum Magazine 39(12) (2002).

${ }^{2}$ B. Yang, J. D. Schaub, S. M. Csutak, D. L. Rogers, and J. C. Campbell, IEEE Photonics Technol. Lett. 15, 745 (2003).

${ }^{3}$ M. K. Emsley, O. I. Dosunmu, and M. S. Ünlü, IEEE J. Sel. Top. Quantum Electron. 8, 948 (2002).

${ }^{4}$ K. Wada, H. Aga, K. Mitani, T. Abe, M. Suezawa, and L. C. Kimerling, "A new approach of photonic band gap formation: Wafer bonding and delamination technique," International Conference-on Solid State Devices and Materials, Hiroshima, Japan, 1998 (unpublished), pp. 382-383. ${ }^{5}$ M. K. Emsley, O. I. Dosunmu, and M. S. Ünlü, IEEE Photonics Technol. Lett. 14, 519 (2002).

${ }^{6}$ B. E. A. Saleh and M. C. Teich, Fundamentals of Photonics (Wiley, New York, 1991).

${ }^{7}$ M. S. Ünlü and S. Strite, Appl. Phys. Rev. 78, 607 (1995).

${ }^{8}$ M. Gökkavas, O. Dosunmu, M. S. Ünlü, G. Ulu, R. P. Mirin, D. H. Christensen, and E. Özbay, IEEE Photonics Technol. Lett. 13, 1349 (2001).

${ }^{9}$ M. S. Ünlü, M. Gökkavas, B. M. Onat, E. Ata, E. Özbay, R. P. Mirin, K. J. Knopp, K. A. Bertness, and D. H. Christensen, Appl. Phys. Lett. 72, 2727 (1998).

${ }^{10}$ N. Biyikli, I. Kimukin, O. Aytür, M. Gökkavas, M. S. Ünlü, and E. Ozbay, IEEE Photonics Technol. Lett. 13, 705 (2001).

${ }^{11}$ D. C. Diaz, C. L. Schow, J. Qi, and J. C. Campbell, Appl. Phys. Lett. 69, 2798 (1996).

${ }^{12}$ J. C. Bean, J. Qi, C. L. Schow, R. Li, H. Nie, J. Schaub, and J. C. Campbell, IEEE Photonics Technol. Lett. 9, 806 (1997).

${ }^{13}$ J. D. Schuab, R. Li, C. L. Schow, and J. C. Campbell, IEEE Photonics Technol. Lett. 11, 1647 (1999).

${ }^{14}$ M. K. Emsley, O. I. Dosunmu, and M. S. Ünlü, IEEE Photonics Technol. Lett. 14, 519 (2002).

${ }^{15}$ C. Maleville, T. Barge, B. Ghyselen, and A. J. Auberton, "Multiple SOI layers by multiple Smart-Cut ${ }^{\circledR}$ transfers," IEEE International SOI Conference, October 2000, pp. 134-135.

${ }^{16}$ Y. Ishikawa, N. Shibata, and S. Fukatsu, Thin Solid Films 321, 234 (1998).

${ }^{17}$ Y. Ishikawa, N. Shibata, and S. Fukatsu, Appl. Phys. Lett. 69, 3881 (1996).

${ }^{18}$ M. K. Emsley and M. S. Ünlü, "Epitaxy-ready reflecting substrates for resonant-cavity-enhanced silicon photodetectors," in Proceedings of IEEE Lasers and Electro-Optics-Society 2000 Annual Meeting, November 2000 (unpublished), Vol. 2, pp. 432-433.

${ }^{19}$ M. K. Emsley, O. I. Dosunmu, and M. S. Ünlü, IEEE J. Sel. Top. Quantum Electron. 8, 948 (2002).

${ }^{20}$ Infineon Tecnologies, "Parallel Optical Links (PAROLI)," http:// www.infineon.com/paroli/, July 2003.

${ }^{21}$ M. S. Ünlü, G. Ulu, and M. Gökkavas, "Resonant cavity enhanced photodetectors," Photodetectors and Fiber-Optics, edited by H. S. Nalwa (Academic, New York, 2001), pp. 98-203. 